International Journal of Bifurcation and Chaos, Vol. 9, No. 11 (1999) 2219-2224

(c) World Scientific Publishing Company

\title{
STUDY OF SYNCHRONIZED MOTIONS IN A ONE-DIMENSIONAL ARRAY OF COUPLED CHAOTIC CIRCUITS
}

\author{
ZBIGNIEW GALIAS and MACIEJ J. OGORZAEEK \\ Department of Electrical Engineering, \\ University of Mining and Metallurgy, Kraków, Poland
}

Received August 18, 1998

\begin{abstract}
We investigate the stability of synchronous motion in an array of bidirectionally coupled electronic circuits. We compute Lyapunov exponents of the generic variational equation associated with directions transversal to the synchronization subspace. Using Lyapunov exponents we derive conditions for the coupling strength for which the stable synchronous solution exists. We also find the limit on the size of the network, which can sustain stable synchronous motion. Theoretical results are compared with the results of numerical experiments.
\end{abstract}

\section{Introduction}

Networks of discretely coupled oscillators provide a versatile model for a variety of phenomena observed in real systems in such areas as physics, biology and medicine [Perez-Munuzuri et al., 1997]. The dynamics of such systems is one of the more lively of studied topics [Kaneko, 1990; Perez-Munñuzuri et al., 1995; Ogorzałek et al., 1995, 1996].

Depending on the dynamics of individual oscillators in the network and the type and strength of coupling between them, a variety of interesting behaviors can be observed, including hyperswitching and clustering [Kaneko, 1990], attractor crowding and various kinds of spatial, temporal or spatiotemporal ordered structures referred to as self-organization [Haken, 1994].

Among various types of dynamical behaviors occurring in coupled systems is the synchronization behavior when some or all cells behave in the same manner (Pyragas [1996] introduced the notions of weak and strong synchronization to distinguish these two cases).

Stability of the synchronous motion becomes a very important problem. In this paper, we study the stability of synchronous motion in a one-dimensional lattice of bidirectionally coupled chaotic circuits.

\section{Dynamics of the Network}

Let us consider a one-dimensional array composed of simple third-order electronic oscillators (Chua's circuits) shown in Fig. 1(a).

The circuits are coupled bidirectionally by means of two resistors cross-connected between the capacitors $C_{1}$ and $C_{2}$ of the neighboring circuits. Every circuit is connected with two nearest neighbors. The dynamics of the one-dimensional lattice composed of $n$ circuits can be described by the following set of ordinary differential equations:

$$
\begin{aligned}
C_{2} \dot{x}_{i}= & -y_{i}+G\left(z_{i}-x_{i}\right) \\
& +G_{1}\left(z_{i-1}-z_{i}\right)+G_{1}\left(z_{i+1}-z_{i}\right), \\
L \dot{y}_{i}= & x_{i} \\
C_{1} \dot{z}_{i}= & G\left(x_{i}-z_{i}\right)-f\left(z_{i}\right) \\
& +G_{1}\left(x_{i-1}-x_{i}\right)+G_{1}\left(x_{i+1}-x_{i}\right),
\end{aligned}
$$

where $i=1,2, \ldots, n$ and the lattice forms a ring $\left(x_{n+1}=x_{1}, z_{n+1}=z_{1}, x_{0}=x_{n}, z_{0}=z_{n}\right)$. 
$f$ is a five-segment piecewise-linear function [compare with Fig. 1(b)]:

$$
\begin{aligned}
f(z)= & m_{2} z+\frac{1}{2}\left(m_{1}-m_{2}\right)\left(\left|z+B_{p_{2}}\right|-\left|z-B_{p_{2}}\right|\right) \\
& +\frac{1}{2}\left(m_{0}-m_{1}\right)\left(\left|z+B_{p_{1}}\right|-\left|z-B_{p_{1}}\right|\right)
\end{aligned}
$$

Observe that Eq. (1) defines a diffusive coupling where the "error" signal for the last variable $z_{i-1}-z_{i}$ and $z_{i+1}-z_{i}$ is injected into the first equation and vice versa. In our study we use typical parameter values for which an isolated circuit generates chaotic oscillations - the "double scroll" attractor $\left(C_{1}=1 / 9 F, C_{2}=1 F, L=1 / 7 H, G=0.7 S\right.$, $m_{0}=-0.8, m_{1}=-0.5, m_{2}=0.8, B_{p_{1}}=1$, $\left.B_{p_{2}}=2\right)$. For the integration of the system the fourth-order Runge-Kutta method was used with the time step $\tau=0.1$.

The setup described above is slightly different from the one used in our previous experiments [Ogorzałek et al., 1995, 1996]. Here we use balanced chaotic circuits, where the value of the resistor connecting capacitors $C_{1}$ and $C_{2}$ in a single circuit is modified. This ensures the existence of a synchronized chaotic solution. If we apply identical initial conditions to every oscillator in the array $\left(x_{i}(0)=x(0), y_{i}(0)=y(0), z_{i}(0)=z(0)\right.$ for $i=1, \ldots, n)$ then all the circuits oscillate synchronously and the equations describing the array

(a)

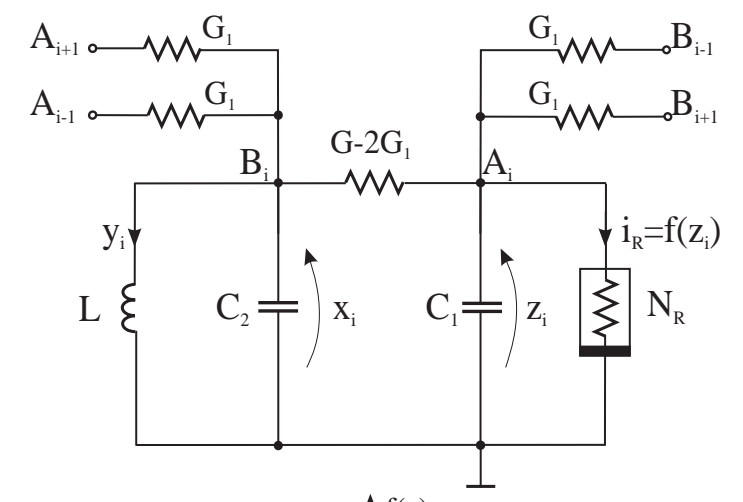

(b)

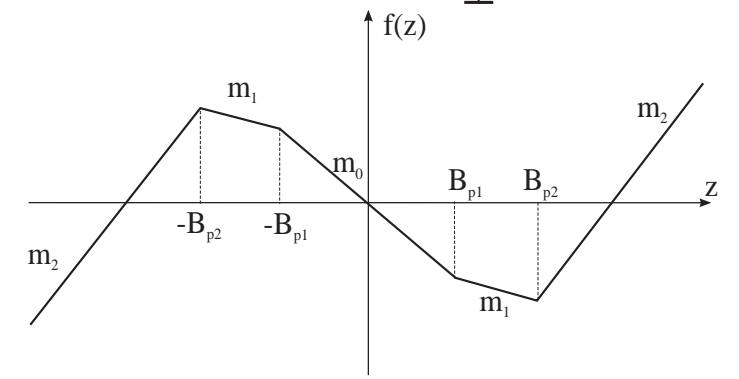

Fig. 1. (a) Nonlinear oscillator and its connection to the neighbors, (b) a five-segment piecewise-linear function. can be written as

$$
\begin{aligned}
C_{2} \dot{x} & =-y+G(z-x), \\
L \dot{y} & =x, \\
C_{1} \dot{z} & =G(x-z)-f(z),
\end{aligned}
$$

where $x_{i}=x, y_{i}=y$ and $z_{i}=z$ for $i=1, \ldots, n$. Hence in the case of equal initial conditions the network as a whole behaves chaotically as a single uncoupled circuit. Our aim in this paper is to investigate the stability of this synchronous solution.

\section{Stability of the Synchronous Motion}

In this section, we investigate the stability of the synchronous motion using Lyapunov exponents. We follow the framework introduced in [Pecora \& Carroll, 1998].

Let $\mathbf{x}_{i}=\left(x_{i}, y_{i}, z_{i}\right)^{T}$ denote the vector of variables of the $i$ th circuit. Let $\boldsymbol{F}$ be the dynamics of the uncoupled circuit, $\boldsymbol{x}=\boldsymbol{F}(\boldsymbol{x})$ as defined by Eq. (3). Then the dynamics of the $i$ th circuit can be written in the following form:

$$
\dot{\mathbf{x}}_{i}=\boldsymbol{F}\left(\boldsymbol{x}_{i}\right)+G_{1} \sum_{j} G_{i j} \boldsymbol{E} \boldsymbol{x}_{j}
$$

where $G_{1}$ is the coupling strength and $\boldsymbol{E}$ is the matrix that is used for coupling. For the bidirectional coupling we consider that the coupling matrix is

$$
\boldsymbol{E}=\left(\begin{array}{ccc}
0 & 0 & 1 / C_{2} \\
0 & 0 & 0 \\
1 / C_{1} & 0 & 0
\end{array}\right)
$$

$\boldsymbol{G}$ is the matrix of couplings between circuits. In our setup we use $G_{i j}=1$ for adjacent oscillators, $G_{i i}=-2$ (this corresponds to the modification of $G$ connecting capacitors $C_{1}$ and $C_{2}$ by $-2 G_{1}$ ) and $G_{i j}=0$ if $|i-j|>1$ (no connection for distant cells):

$$
\boldsymbol{G}=\left(\begin{array}{rrrrrr}
-2 & 1 & 0 & \ldots & 0 & 1 \\
1 & -2 & 1 & \ldots & 0 & 0 \\
0 & 1 & -2 & \ldots & 0 & 0 \\
\vdots & \vdots & \vdots & \ddots & \vdots & \vdots \\
0 & 0 & 0 & \ldots & -2 & 1 \\
1 & 0 & 0 & \ldots & 1 & -2
\end{array}\right) .
$$


For the network composed of $n=2$ circuits the self-coupling coefficient is $G_{i i}=-1$ (each circuit is connected with one circuit only and in order to balance the circuit we have to modify the value of the conductance $G$ by $-G_{1}$ ). Hence in this case, the matrix $\boldsymbol{G}$ has the form:

$$
\boldsymbol{G}=\left(\begin{array}{rr}
-1 & 1 \\
1 & -1
\end{array}\right)
$$

In all cases $(n=2, n \geq 3)$ the $\operatorname{sum} \sum_{j} G_{i j}=0$ and hence the synchronization manifold in invariant (if $\mathbf{x}_{i}=\mathbf{x}_{j}$ for all $i, j$ the last term in Eq. (4) disappears).

Let us create the variational equation of Eq. (4) and diagonalize $\mathbf{G}$ obtaining a block diagonalized variational equation with each block having the form (for details see [Pecora \& Carroll, 1998]):

$$
\dot{\xi}_{k}=\left(D \mathbf{F}+G_{1} \gamma_{k} \mathbf{E}\right) \xi_{k},
$$

where $\gamma_{k}$ are eigenvalues of $\mathbf{G}(k=0, \ldots, N-1)$. The above equation for $k=0$ is the variational equation for the synchronization manifold. Other eigenvalues correspond to transverse eigenvectors.

The idea, introduced in [Pecora \& Carroll, 1998], is to compute the maximum Lyapunov exponent for the generic variational equation

$$
\dot{\xi}=(D \mathbf{F}+\gamma \mathbf{E}) \xi,
$$

as a function of $\gamma$. In the general case, Eq. (9) should be solved for $\gamma$ from the complex plane. In our case, however, as the matrix $\mathbf{G}$ has only real eigenvalues it suffices to solve it for real line only.

We have computed the maximum Lyapunov exponent of the generic variational equation for 60 equidistant points from the interval $(-2,1)$. The results are shown in Fig. 2.

In order to use the solution of the variational equation plotted in Fig. 2 for the investigation of stability of synchronous motion let us assume that we have $n$ oscillators in the array and the coupling strength is $G_{1}$. First we compute the eigenvalues $\gamma_{k}$ of G. For $n=2$ the eigenvalues are $\gamma_{0}=0$, $\gamma_{1}=2$. For $n \geq 3$ they can be computed as $\gamma_{k}=$ $-4 \sin ^{2} \pi k / n$. We pick up the eigenvalues associated with transverse eigenvectors $\left(\gamma_{1}, \ldots, \gamma_{n-1}\right)$. The synchronous motion is stable if $\lambda_{\max }$ is negative for $\gamma=G_{1} \gamma_{k}$, where $k=1, \ldots, n-1$. We can read this information from Fig. 2.

Now we derive conditions for $G_{1}$ ensuring stable synchronous state and find the array sizes for

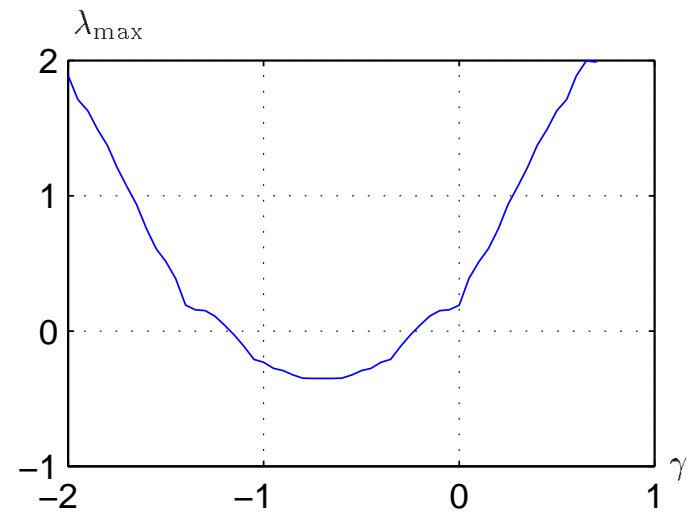

Fig. 2. Maximum Lyapunov exponent $\lambda_{\max }$ of the generic variational equation for real $\gamma$.

which it is possible to obtain stable synchronous solution. Let us assume that there exist an interval of $\gamma$ for which the maximum Lyapunov exponent of Eq. (9) is negative. From Fig. 2 one can see that this assumption is fulfilled. Let us denote the ends of this interval by $\alpha$ and $\beta$ (with $\alpha<\beta<0$ ). We have estimated that in our case $\alpha \approx-1.171$ and $\beta \approx-0.229$.

The conditions for the stability of synchronous motion are $\alpha<G_{1} \gamma_{k}<\beta$ for $k=1, \ldots, n-1$. Using the formulas for $\gamma_{k}$ one can easily obtain the range of $G_{1}$, for which synchronization is possible:

$$
G_{1} \in\left(\frac{-\beta}{2}, \frac{-\alpha}{2}\right) \quad \text { for } n=2,
$$$$
G_{1} \in\left(\frac{-\beta}{4 \sin ^{2} \frac{\pi}{n}}, \frac{-\alpha}{4}\right) \quad \text { for even } n \geq 4,
$$

$G_{1} \in\left(\frac{-\beta}{4 \sin ^{2} \frac{\pi}{n}}, \frac{-\alpha}{4 \sin ^{2} \frac{(n-1) \pi}{2 n}}\right) \quad$ for odd $n \geq 3$.

For a given network size $n$ synchronization is possible if the above intervals are not empty. For $n=2$ the interval $(-\beta / 2,-\alpha / 2)$ is nonempty if $-\beta<-\alpha$, which is true in our case. Now let us consider $n \geq 3$. The intervals in Eq. (10) are not empty if

$$
\begin{gathered}
\sin ^{2} \frac{\pi}{n}>\frac{\beta}{\alpha} \quad \text { for even } n, \\
\frac{\sin ^{2} \frac{\pi}{n}}{\sin ^{2} \frac{(n-1) \pi}{2 n}}>\frac{\beta}{\alpha} \quad \text { for odd } n
\end{gathered}
$$


Table 1. Coupling strength $G_{1}$ for which $\lambda_{\max }$ is negative for $G_{1} \gamma_{i}$, $i=1, \ldots, n-1$. For these values the existence of stable synchronized state is possible.

\begin{tabular}{cc}
\hline$n$ & $G_{1}$ \\
\hline 2 & $(0.1145,0.5855)$ \\
3 & $(0.0763,0.3903)$ \\
4 & $(0.1145,0.2928)$ \\
5 & $(0.1657,0.3237)$ \\
6 & $(0.2290,0.2928)$ \\
7 & $(0.3041,0.3080)$ \\
\hline
\end{tabular}

One can easily obtain the following conditions:

$$
\begin{gathered}
n<\frac{\pi}{\arcsin \sqrt{\beta / \alpha}} \approx 6.86 \text { for even } n, \\
n<\frac{\pi}{2 \arcsin 0.5 \sqrt{\beta / \alpha}} \approx 7.05 \text { for odd } n .
\end{gathered}
$$

Thus, synchronization is possible for $n=$ $2, \ldots, 7$. In Table 1 , we collect the values of the coupling strength $G_{1}$ for which the maximum Lyapunov exponent of Eq. (9) is negative for $G_{1} \gamma_{i}$, $i=1, \ldots, n-1$. They were obtained using Eq. (10).

\section{Computer Simulations}

In this section we compare theoretical predictions with the results of computer experiments.

In order to test the stability of a particular solution one can perturb this solution by a random additive signal with a small amplitude and observe the steady-state behavior of the system. If the system converges to the solution under consideration one claims that the solution is stable.

We have performed such an experiment for $n=2, \ldots, 7$ and different $G_{1}$. The value of $G_{1}$ was modified from 0 to 0.6 with the step 0.005 . The results are shown in Fig. 3. As a solid line we plot theoretical predictions from Table 1. In experiments the chaotic synchronous solution is perturbed by a random additive signal of amplitude 0.0001 (result plotted below the solid line) and 0.01 (result plotted above the solid line). Results of computer simulations are plotted as dots. The dot is plotted if after time $t=1000$ the state is still synchronous.

One can clearly see that the results of experiments with smaller amplitude of perturbation are closer to the theoretical results. This corresponds to the fact that using Lyapunov exponents we can obtain only local information along the synchronization subspace. If the initial point is far from the synchronization subspace we cannot predict the behavior of the system using Lyapunov exponents. Another problem is that the negativity of Lyapunov exponents is only a necessary condition for the existence of stable synchronous solution.

It is interesting to note that for $n=7$ we have not found $G_{1}$ with experimentally stable synchronous solution. This will be discussed later.

Now let us discuss several examples. In Fig. 4 we plot the steady-state behavior for $n=3$ and four different coupling values $G_{1}=0.05,0.1,0.35,0.4$. For each case we plot $y_{i}$ versus $y_{i+1}$. Hence we can clearly see whether the neighboring circuits are synchronized. One can observe that for $G_{1}=0.1$

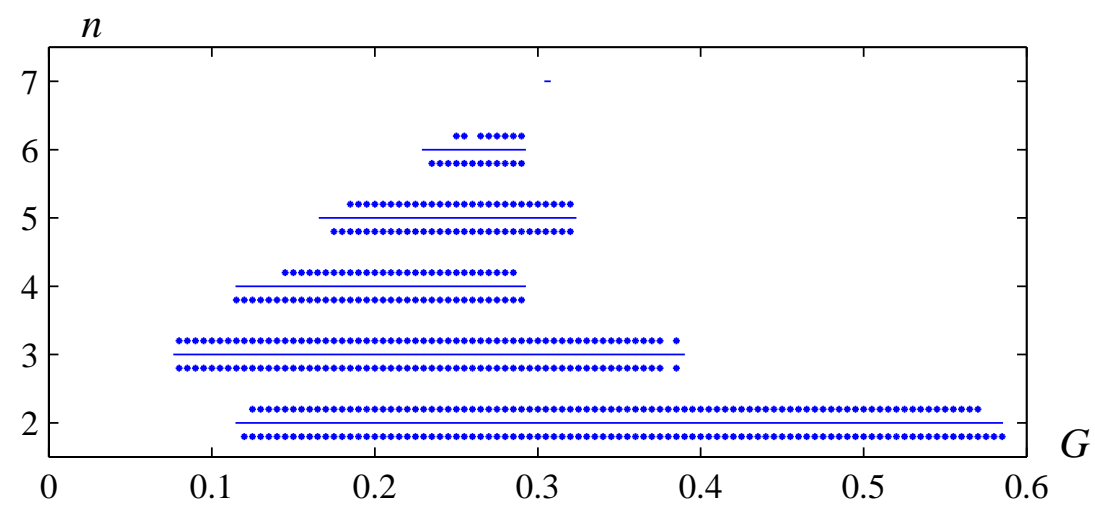

Fig. 3. Theoretical versus simulation results for the network size $n=2, \ldots, 7$. Solid line - theoretical prediction based on conditional Lyapunov exponents, lower and upper dots - synchronous behavior in the steady-state observed in computer simulations after perturbation of the synchronous state by random signals of amplitude 0.0001 and 0.01 , respectively. 


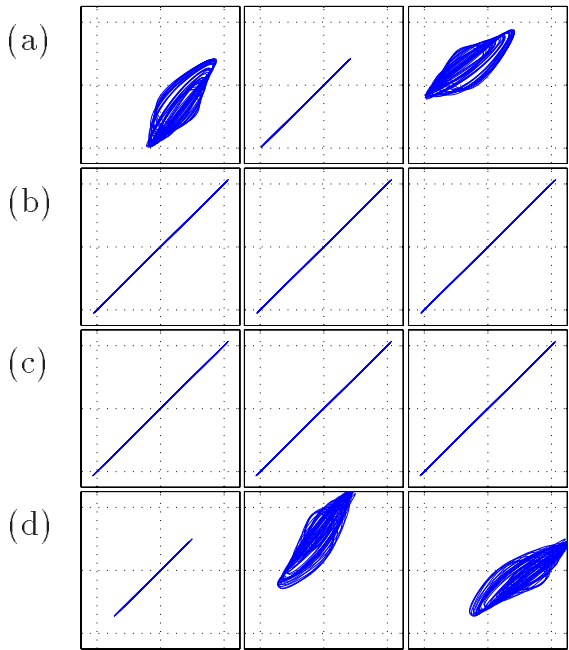

Fig. 4. Steady-state of perturbed synchronous motion in an array composed of $n=3$ circuits for different coupling strengths: (a) $G_{1}=0.05$, (b) $G_{1}=0.1$, (c) $G_{1}=0.35$, (d) $G_{1}=0.4$. In each row, we plot $y_{i}$ versus $y_{i+1}$ for $i=1,2,3$.

and $G_{1}=0.35$ the steady-state is synchronous, while for the other two cases the steady-state is not synchronous. Although it is not synchronous globally, one can see that in both cases there exists a cluster of two circuits oscillating synchronously. Results observed numerically are in good agreement with the theoretical prediction, from which it follows that the synchronous state is stable for $G_{1} \in(0.0763,0.3903)$ (compare Table 1 and Fig. 3$)$.

We obtain similar results for $n=5$. For $G_{1}=0.17$ and $G_{1}=0.32$ we observe stability of synchronous behavior, while for $G_{1}=0.15$ and $G_{1}=0.325$ the synchronous mode is not stable (see Fig. 5). The experimental results agree very well with theoretical predictions (compare Table 1). Another interesting phenomenon is the existence of clusters composed of two circuits oscillating synchronously. There is one cluster for $G_{1}=0.15$ and two clusters for $G_{1}=0.325$. One of them is not visible as it is composed of circuits 3 and 5, which are not neighbors.

Finally let us consider the network composed of seven oscillators. In this case, the interval of coupling strength with stable synchronous motion is very narrow $G_{1} \in(0.304,0.308)$. We choose two values of coupling coefficients: $G_{1}=0.30$ and $G_{1}=0.306$. The trajectory of the system after time $T=1000$ is shown in Figs. 6(a) and 6(b). In the steady-state, the system is not in the synchronous mode. This is in contrast to the theoretical predictions, as for the second case we expect the synchronization behavior. The escape from synchronization manifold is very slow. For $G_{1}=0.30$ we observe loss of synchronous behavior after $T \approx 400$. For $G_{1}=0.306$ the escape time is even longer: $T \approx 800$. We believe that the reason for this disagreement is small noise, coming from the integration procedure, that causes desynchronization bursts. The stability of synchronous behavior is not robust (the maximum Lyapunov exponents corresponding to transversal directions is negative but very close to zero). We have repeated the experiment using smaller integration step ( $\tau=0.02$ instead of $\tau=0.1)$. The results are shown in Fig. 6(c).

(a)

(b)

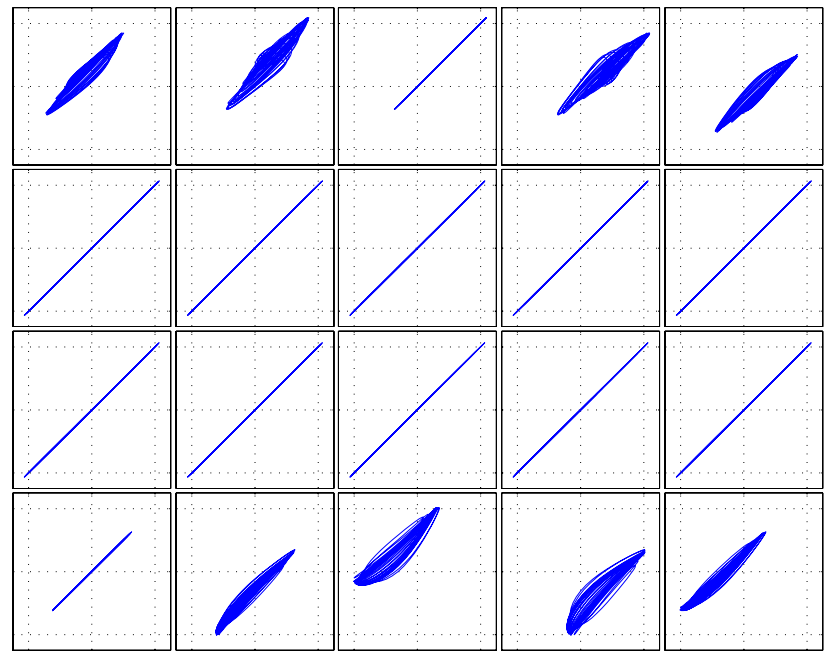

Fig. 5. Steady-state of perturbed synchronous motion in an array composed of $n=5$ circuits for different coupling strength: (a) $G_{1}=0.15$, (b) $G_{1}=0.17$, (c) $G_{1}=0.32$, (d) $G_{1}=0.325$. 


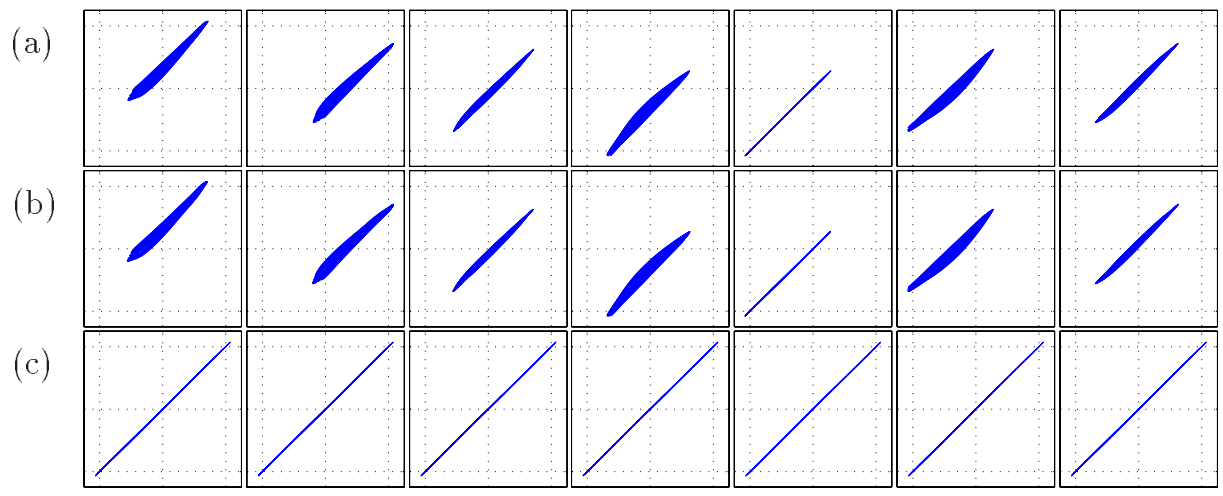

Fig. 6. Steady-state of disturbed synchronous motion in an array composed of $n=7$ circuits for different coupling strength: (a) $G_{1}=0.30$, (b) $G_{1}=0.306$, time step $\tau=0.1$ (c) $G_{1}=0.306$, time step $\tau=0.02$.

After a very long time $T=10000$ one still observes synchronization behavior.

\section{Conclusions}

In this paper we have investigated the stability of synchronous solution of a one-dimensional array of bidirectionally coupled chaotic circuits. We have found the upper limit on the size of the network, that can sustain stable synchronous motions. For different array sizes we have found the ranges of the coupling strength, for which the synchronous motion is stable. We have confirmed that the theoretical predictions of the existence of the stable synchronous solution compare very well with the results of computer simulations. We would like to stress that the study of synchronization properties based on Lyapunov exponents presented in this paper gives local information in the neighborhood of the synchronous state only. Many other attractors may exist and in fact, for each coupling strength considered we have observed an abundance of attractors without the synchronization property.

\section{Acknowledgments}

This research has been supported by the University of Mining and Metallurgy, Kraków, Poland, research fund 11.120.460. We would like to thank L. Pecora for fruitful discussions.

\section{References}

Haken, H. [1994] "Synergetics; from pattern formation to pattern analysis and pattern recognition," Int. J. Bifurcation and Chaos 4, 1069-1083.

Kaneko, K. [1990] "Clustering, coding, switching, hierarchical ordering and control in a network of chaotic elements," Physica D41, 137-172.

Ogorzałek, M., Galias, Z., Dạbrowski, A. \& Dạbrowski, W. [1995] "Chaotic waves and spatio-temporal patterns in large arrays of doubly-coupled Chua's circuit," IEEE Trans. Circuits Syst. 42(10), 706-714.

Ogorzałek, M., Galias, Z., Dąbrowski, A. \& Dąbrowski, W. [1996] "Wave propagation, pattern formation and memory effects in large arrays of interconnected chaotic circuits," Int. J. Bifurcation and Chaos 6(10), 1859-1871.

Pecora, L. \& Carroll, T. [1998] "Master stability functions for synchronized coupled systems," Phys. Rev. Lett. 80(10), 2109-2112.

Perez-Muñuzuri, A., Perez-Muñuzuri, V., GomezGesteira, M., Chua, L. \& Perez-Villar, V. [1995] "Spatio-temporal structures in discretely-coupled arrays of nonlinear circuits: A review," Int. J. Bifurcation and Chaos 5, 17-50.

Perez-Muñuzuri, V., Perez-Villar, V., Chua, L. \& Marcus, M. (eds.) [1997] Discretely-Coupled Dynamical Systems, World Scientific Series on Nonlinear Science, Series B, Vol. 9 (World Scientific, Singapore).

Pyragas, K. [1992] "Continuous control of chaos by selfcontrolling feedback," Phys. Lett. A170, 421-428.

Pyragas, K. [1996] "Weak and strong synchronization of chaos," Phys. Rev. E54, 4508-4511. 